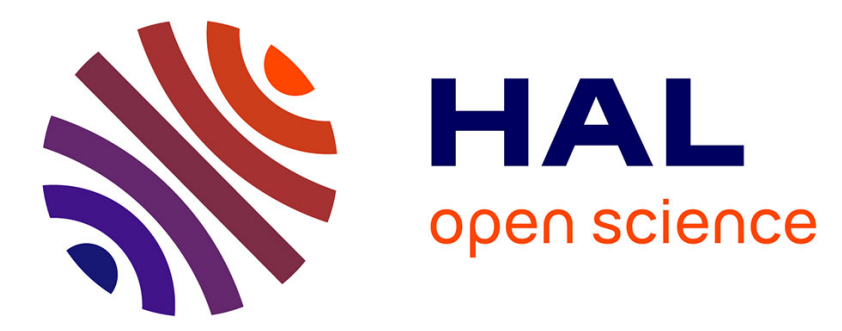

\title{
Improved sensitivity at synchrotrons using edge illumination X-ray phase-contrast imaging
}

P. C. Diemoz, M. Endrizzi, C. E. Zapata, A. Bravin, R. D. Speller, I. K. Robinson, A. Olivo

\section{> To cite this version:}

P. C. Diemoz, M. Endrizzi, C. E. Zapata, A. Bravin, R. D. Speller, et al.. Improved sensitivity at synchrotrons using edge illumination X-ray phase-contrast imaging. Journal of Instrumentation, 2013, 8, 8 p. 10.1088/1748-0221/8/06/C06002 . hal-01573943

\section{HAL Id: hal-01573943 \\ https://hal.science/hal-01573943}

Submitted on 11 Aug 2017

HAL is a multi-disciplinary open access archive for the deposit and dissemination of scientific research documents, whether they are published or not. The documents may come from teaching and research institutions in France or abroad, or from public or private research centers.
L'archive ouverte pluridisciplinaire HAL, est destinée au dépôt et à la diffusion de documents scientifiques de niveau recherche, publiés ou non, émanant des établissements d'enseignement et de recherche français ou étrangers, des laboratoires publics ou privés. 


\title{
Improved sensitivity at synchrotrons using edge illumination X-ray phase-contrast imaging
}

\author{
P.C. Diemoz, ${ }^{a, d, 1}$ M. Endrizzi, ${ }^{a}$ C.E. Zapata, ${ }^{a, d}$ A. Bravin, ${ }^{b}$ R.D. Speller, ${ }^{a}$ \\ I.K. Robinson ${ }^{c, d}$ and A. Olivo ${ }^{a, d}$ \\ ${ }^{a}$ Department of Medical Physics and Bioengineering, University College London, \\ Malet Place, Gower Street, WC1E 6BT London, United Kingdom \\ ${ }^{b}$ European Synchrotron Radiation Facility, \\ 6 rue J. Horowitz, BP 220, 38043 Grenoble, France \\ ${ }^{c}$ London Centre for Nanotechnology, \\ 17-19 Gordon Street, WC1H OAH London, United Kingdom \\ ${ }^{d}$ Research Complex at Harwell, \\ Harwell Oxford Campus, OX11 OFA Didcot, United Kingdom \\ E-mail: p.diemoz@ucl.ac.uk
}

\begin{abstract}
The application of the X-ray phase-contrast 'edge illumination' principle to the highly coherent beams available at synchrotron radiation facilities is presented here. We show that, in this configuration, the technique allows achieving unprecedented angular sensitivity, of the order of few nanoradians. The results are obtained at beamlines of two different synchrotron radiation facilities, using various experimental conditions. In particular, different detectors and X-ray energies $(12 \mathrm{keV}$ and $85 \mathrm{keV}$ ) were employed, proving the flexibility of the method and the broad range of conditions over which it can be applied. Furthermore, the quantitative separation of absorption and refraction information, and the application of the edge illumination principle in combination with computed tomography, are also demonstrated. Thanks to its extremely high phase sensitivity and its flexible applicability, this technique will both improve the image quality achievable with X-ray phasecontrast imaging and allow tackling areas of application which remain unexplored until now.
\end{abstract}

KEYWORDS: Image reconstruction in medical imaging; X-ray radiography and digital radiography (DR); Computerized Tomography (CT) and Computed Radiography (CR); Data processing methods

\footnotetext{
${ }^{1}$ Corresponding author.
} 


\section{Contents}

1 Introduction 1

2 The edge illumination principle 2

3 Experimental results $\quad 4$

4 Conclusions 6

\section{Introduction}

X-ray phase-contrast imaging (XPCi), as opposed to absorption-based methods, does not rely only on the beam attenuation induced by the sample, but it also exploits refraction/interference effects to enhance the image contrast. The improvement compared with conventional absorption-based methods is very high in particular for the range of X-ray energies used in radiology (10-100 keV) and for materials made of low atomic number elements, as those characteristic of biological soft tissues. Since in this case refraction/interference effects can be detectable even when the absorption signal is negligible, XPCi methods can provide largely increased sensitivity to weakly absorbing object details.

Several XPCi techniques have been developed and applied [1-6]. Much research effort has been recently dedicated, in particular, to three XPCi methods: free-space propagation (FSP) [2], analyzer-based imaging (ABI) [3] and grating interferometry (GI) [4], which have demonstrated excellent results in terms of tissue discrimination and characterization, especially in the field of biomedical imaging, for a wide range of different applications [7-10].

In this work, we investigate the application of a different technique, which is based on the edge illumination (EI) principle. This was first proposed at the Elettra synchrotron radiation (SR) facility (Trieste, Italy) in the late nineties [11], as a method to finely analyze the beam refraction introduced by the sample. The idea was to reproduce, by means of a simpler setup, the signal obtainable with the ABI technique, which exploits the angular dependence of diffraction from a perfect crystal to sense the beam refraction. The application of the EI principle with extended (up to at least $100 \mu \mathrm{m}$ focal spot) and polychromatic sources was then demonstrated theoretically and experimentally [12-14]. In this case, an appropriate pair of pre-sample and detector masks is used to replicate the EI principle over the entire field of view of an area detector, and the technique is thus referred to as coded-aperture XPCi.

In addition to being efficiently implementable with conventional sources, we recently demonstrated that EI enhances the phase sensitivity compared with other XPCi methods if used with highly coherent beams provided by SR facilities. We proved that, at very large X-ray energies where the signal is generally very small, EI outperformed FSP by providing an image contrast up to twenty times larger [15]. We show here how extremely high angular sensitivity can be achieved 

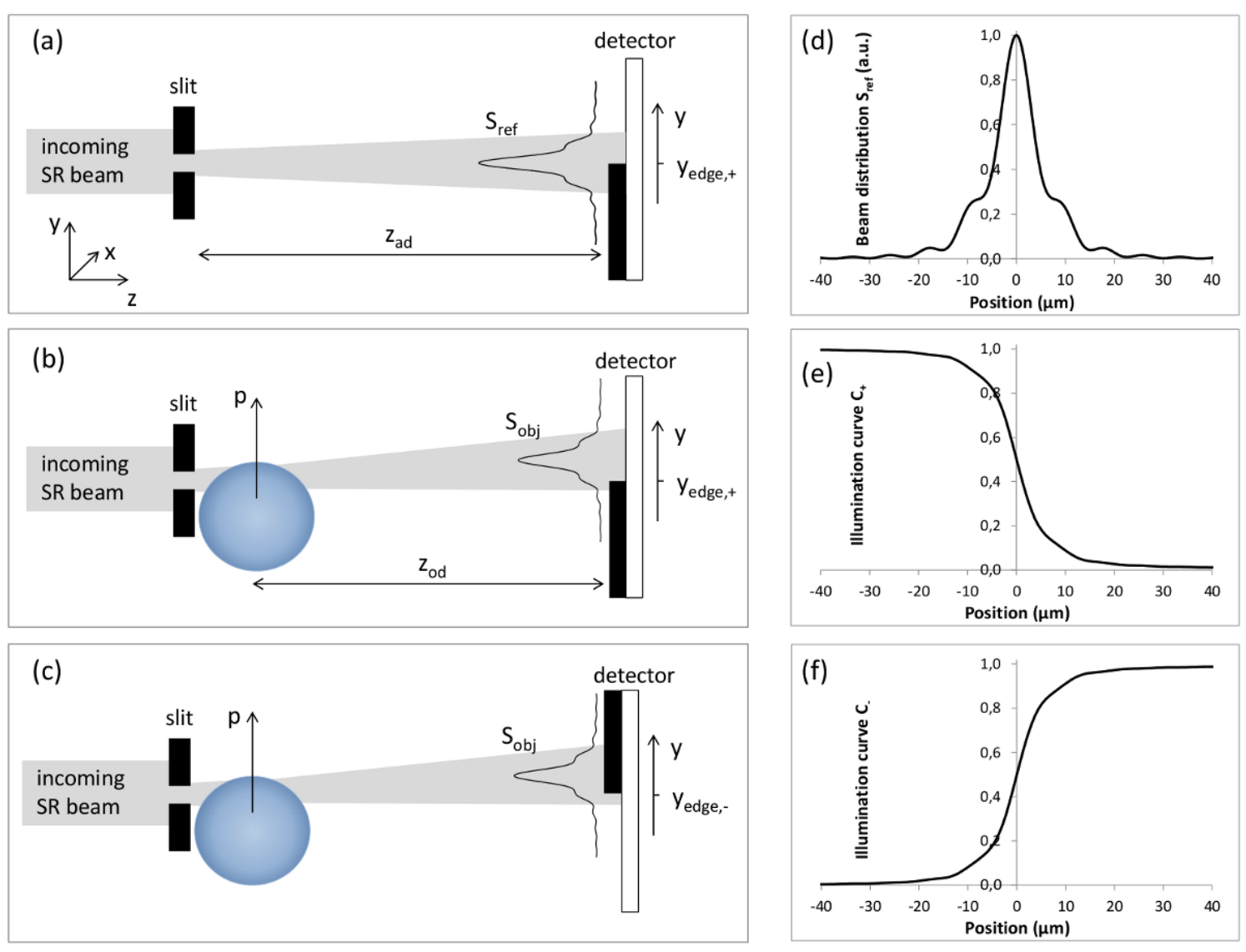

Figure 1. Illustration of the EI principle. (a) Diagram of the EI setup, in the case of a detector edge realising the partial illumination condition by covering the lower part of the beam. (b) EI setup with a refracting sample in the collimated beam, in the case of a detector edge covering the lower part of the beam, or (c) the upper part of the beam. (d) Distribution of the reference beam incident onto the absorbing edge, by considering the setup used at the ID17 beamline of the ESRF (see text for details). (e) Illumination curve corresponding to beam in (d), in the case of an edge covering the lower or (f) the upper part of the beam.

under various experimental conditions, and in particular at completely different (very low and very high, for radiology) X-ray energies. The possibility of extracting quantitative information about the absorption and refraction properties of the sample, and the applicability of the method to computed tomography, are also presented.

\section{The edge illumination principle}

The EI principle is schematized in figure 1, where the case of a parallel and monochromatic SR beam is considered. The beam is collimated in one direction $(y)$ by means of slits and aligned with an absorbing edge placed in front of the detector, so that part of the beam is directly incident on a line of detector pixels while part is stopped by the edge (figure 1a). When an object is inserted into the collimated beam, the photons can be deviated upwards or downwards, increasing or decreasing the part of the beam reaching the detector, and thus the detected intensity (figure 1b). An image of the sample is obtained by scanning it through the beam in the direction $y$ perpendicular to the edge. 
The boundaries of details inside the sample (where the largest refraction is observed) will be highlighted in the image by either dark or white fringes, according to the refraction direction; by cutting the opposite side of the beam with the absorbing edge, this fringe contrast is reversed (figure 1c). Being sensitive to the same physical quantity (the contrast is to first approximation proportional to the refraction angle in one direction, $\Delta \theta_{y}$ ), the obtained image and the corresponding signal are analogous to those achievable with the $\mathrm{ABI}$ and GI techniques.

Let us indicate with $S_{\text {ref }}$ the beam distribution (i.e. the number of photons per unit length in the $y$ direction) incident onto the detector edge in the absence of the object. In a ray-tracing model, $S_{\text {ref }}$ can be expressed as the convolution of the projection of the pre-sample slits onto the detector plane and the projected source distribution (i.e. as the blurred shadow of the pre-sample slits onto the detector). However, in the case of highly spatially and temporally coherent SR, diffraction effects cannot be neglected, and the rigorous wave optics formalism must be employed to determine the distribution of the beam impinging on the detector edge [16]. An example of the beam distribution obtainable in the setup employed at the ID17 beamline of the ESRF is presented in figure 1d, where the considered vertical source size is $24 \mu \mathrm{m}$, the source-to-slits distance $z_{s a}=145 \mathrm{~m}$, the slits-to-detector distance $z_{a d}=10.3 \mathrm{~m}$ and the slits aperture size is $20 \mu \mathrm{m}$.

The intensity recorded by the detector in the case of an edge, set at position $y_{\text {edge },+}$, covering the lower part of the beam can be expressed as:

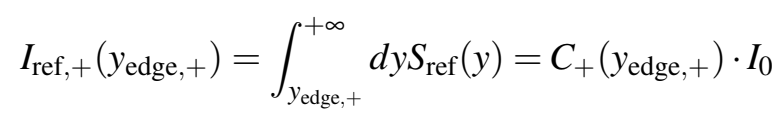

where $I_{0}$ is the total number of photons incident on the sample and $C_{+} \equiv \int_{y_{\text {edge },+}^{+}}^{+\infty} d y S_{\text {ref }}(y) / I_{0}$ is the illumination curve, sometimes referred to as rocking curve due to the analogy with the ABI technique. The illumination curve $C_{+}$corresponding to the beam distribution in figure $1 \mathrm{~d}$ is reported in figure 1e.

When the sample is inserted, at a distance $z_{o d}$ upstream the detector, the beam is shifted in the $y$ direction of the quantity $z_{o d} \cdot \Delta \theta_{y}$ as an effect of the sample refraction, and attenuated due to absorption:

$$
S_{\text {obj }}(y, p)=T(p) \cdot S_{\text {ref }}\left(y-z_{o d} \cdot \Delta \theta_{y}(p)\right)
$$

where $p$ is the position in the sample and $T$ indicates the corresponding X-ray transmission. Note that equation (2.2) is strictly valid only if the absorption and the refraction angle are approximately constant within the size of the pre-sample aperture. The intensity recorded by the detector is therefore:

$$
I_{\mathrm{obj},+}\left(y_{\text {edge },+}, p\right)=T(p) I_{0} \cdot C_{+}\left(y_{\text {edge },+}-z_{o d} \cdot \Delta \theta_{y}(p)\right)
$$

In the case of an absorbing edge cutting, instead of the lower, the upper part of the beam (figure 1c), the intensity detected without the object can be expressed as:

$$
I_{\text {ref },-}\left(y_{\text {edge },-}\right)=\int_{-\infty}^{y_{\text {edge, }-}} d y S_{\text {ref }}(y)=C_{-}\left(y_{\text {edge },-}\right) \cdot I_{0}
$$

where the illumination curve is here defined as $C_{-} \equiv \int_{-\infty}^{y_{\text {edge, }}} d y S_{\text {ref }}(y) / I_{0}$. The intensity detected with the object in the beam is then:

$$
I_{\mathrm{obj},-}\left(y_{\text {edge },-}, p\right)=T(p) I_{0} \cdot C_{-}\left(y_{\text {edge },-}-z_{o d} \cdot \Delta \theta_{y}(p)\right)
$$



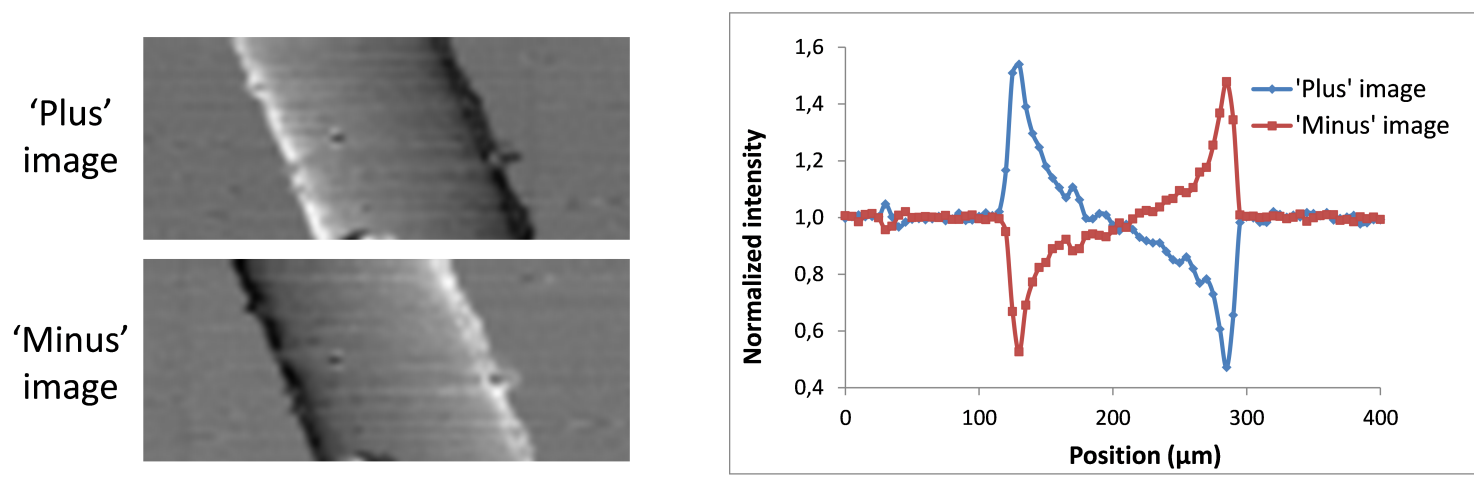

Figure 2. EI images of a $165 \mu \mathrm{m}$ diameter PEEK filament in water, obtained at Diamond (I13 beamline) at an energy of $12 \mathrm{keV}$ (see details in text). The two images were acquired respectively with the detector edge covering the lower ('plus' image) or the upper ('minus' image) half of the beam (note that the presented images have been rotated by 90 degrees: the pre-sample slits and detector edge are in fact horizontal, and the system is thus sensitive to refraction in the vertical direction). The corresponding intensity profiles across the filament are reported on the right.

The illumination curve $C_{-}$corresponding to the beam distribution in figure $1 \mathrm{~d}$ is shown in figure $1 \mathrm{f}$. From the definitions of $C_{+}$and $C_{-}$, and from figures $1 \mathrm{~b}$ and $1 \mathrm{c}$, it is evident that $C_{-}\left(y_{\text {edge }}-z_{o d}\right.$. $\left.\Delta \theta_{y}\right)=1-C_{+}\left(y_{\text {edge }}-z_{o d} \cdot \Delta \theta_{y}\right)$. As a result, the detected refraction signals will be perfectly reversed in the two cases: positive (or negative) signals in the 'plus' configuration will become negative (or positive) signals in the 'minus' one.

\section{Experimental results}

We present the results obtained at two different SR facilities, in order to show how extremely high sensitivity is achievable with this technique in very different experimental conditions. In particular, the experiments were carried out at both very high and very low X-ray energies, demonstrating that the method can be applied over a very broad range of energies and thus for a variety of different samples and applications.

In figure 2, we report the images of a polyetheretherketone (PEEK) filament (diameter $165 \mu \mathrm{m}$ ) immersed in water, obtained at the I13 beamline (coherence branch) of the Diamond SR facility (Didcot, U.K.). An X-ray energy of $12 \mathrm{keV}$ was used. The nominal vertical source size is $15 \mu \mathrm{m}$, the source-to-slits distance was $z_{s a}=220 \mathrm{~m}$, the slits-to-object distance $z_{a o}=9 \mathrm{~cm}$, the object-to-detector distance $z_{o d}=14.69 \mathrm{~m}$ and the aperture size was $20 \mu \mathrm{m}$. The object scan step in the direction transversal to the edge was $5 \mu \mathrm{m}$, and a MEDIPIX detector, with a pixel size of $55 \mu \mathrm{m}$, was used for the image acquisition [17]. Both the slits and the detector edge were oriented horizontally, so that the setup is sensitive to the component of the refraction angle parallel to the vertical direction $y$ (note that the presented images have been rotated by 90 degrees, so that the sensitivity appears to be in the horizontal direction).

The first image in figure 2 was acquired with the edge cutting the lower half of the beam ('plus' image, setup configuration presented in figures 1a and 1b), while the second was obtained with the edge cutting the upper half ('minus' image, see figure 1c). On the right, intensity profiles 

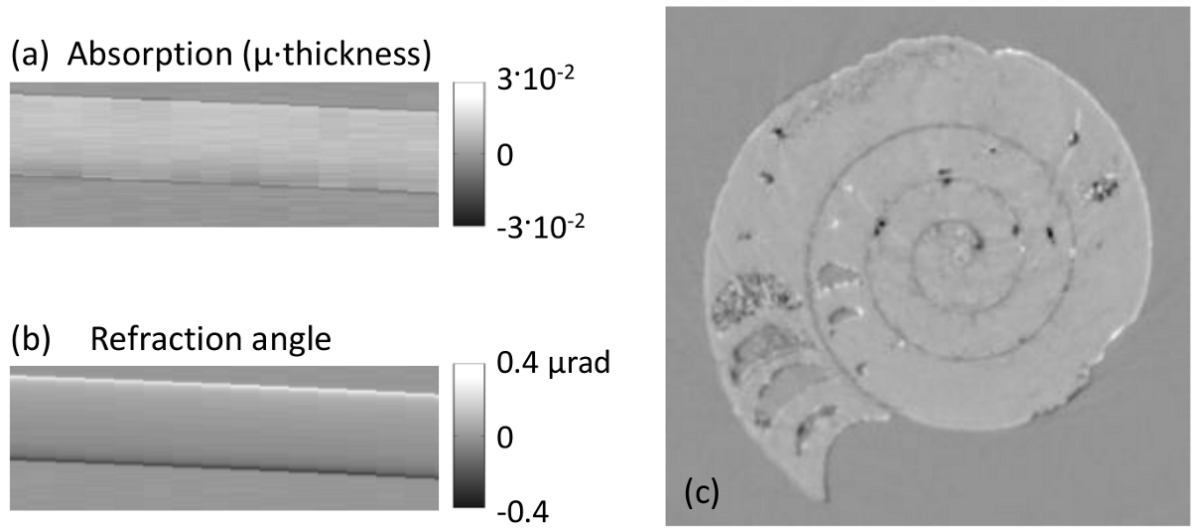

\section{(b) Refraction angle}

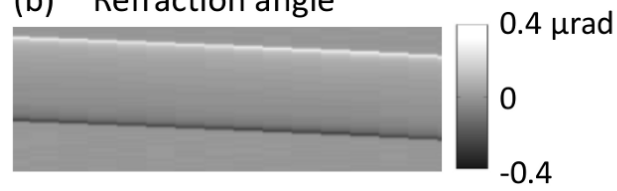

(c)

Figure 3. Results obtained at the ESRF (ID17 beamline) at an energy of $85 \mathrm{keV}$ (see details in text). (a) Extracted absorption and (b) refraction images of a $450 \mu \mathrm{m}$ thick PEEK filament. (c) CT slice of an ammonite sample, reconstructed by using the mixed absorption-refraction method [23].

across the filament in the two images are reported. The left and right edges of the object feature opposite signals, since the X-rays are refracted in opposite directions at the two object positions. Furthermore, as expected from the theory (see section 2), the signal is perfectly reversed in the two images acquired at the 'plus' and 'minus' positions of the edge. Despite the fact that the PEEK filament is immersed in water $\left(\delta_{\mathrm{PEEK}}=1.90 \cdot 10^{-6}\right.$ and $\delta_{\text {wat }}=1.60 \cdot 10^{-6}$, at $\left.12 \mathrm{keV} \mathrm{[18]}\right)$, the measured contrast (i.e. the difference between the maximum and the minimum of the object profile, divided by the mean) is higher than $100 \%$.

A separate experiment was carried out at the ID17 biomedical beamline of the ESRF (Grenoble, France). In this case, a much higher X-ray energy of $85 \mathrm{keV}$ was used. The nominal vertical source size is $24 \mu \mathrm{m}$, the opening of the pre-sample slits was $20 \mu \mathrm{m}, z_{s a}=145 \mathrm{~m}, z_{a o}=4.3 \mathrm{~m}$ and $z_{o d}=6.0 \mathrm{~m}$. Images were acquired with a Germanium detector of $350 \mu \mathrm{m}$ pixel size [19].

Filaments of different size and composition were imaged, and quantitative absorption and refraction maps were extracted. They were calculated by combining the 'minus' and 'plus' images by using a new quantitative method, specifically developed for the application to the highly coherent beams provided by SR facilities [20]. The absorption and refraction images obtained for a $450 \mu \mathrm{m}$ PEEK wire are presented in figures $3 \mathrm{a}-\mathrm{b}$ (the used scan step was $20 \mu \mathrm{m}$ ). Both images contain very small contribution from noise. In order to quantify it, 30 background regions of $40 \times 40$ pixels $^{2}$ were selected in the refraction image, the standard deviation of the pixel values calculated for each region and the results averaged. This calculation provided a value for the noise in the refraction image lower than $2 \mathrm{nrad}$, which is about one order of magnitude smaller than previously published results for grating interferometry [21,22]. This means that very high phase sensitivity is achieved by this setup, since extremely small refraction angles, down to about $2 \mathrm{nrad}$, are detectable. Similar calculations were performed for the setup used at the I13 beamline of Diamond, showing values of angular resolution still very high but slightly lower than those observed at the ESRF, with achieved levels of noise of the order of $15 \mathrm{nrad}$. We expect this to be due to a combination of factors: lower photon statistics used for the image acquisition compared to the ESRF experiment, broadening of the beam at lower energies (leading to slightly decreased sensitivity to beam angular deviations), inhomogeneity in the detector pixels response and thermal load on the monochromator. There- 
fore, we expect even higher sensitivities once the fine adjustements are implemented on a beamline which, at the time of the experiment, had just been taken out of its commissioning phase.

The application of the EI technique to computed tomography (CT) was also demonstrated. Figure $3 \mathrm{c}$ shows a CT slice of an ammonite fossil, obtained using the setup at the ID17 beamline described above and rotating the sample around the axis orthogonal to the plane containing the beam. This is a highly absorbing sample characterized by a considerable iron content, which thus requires very high X-ray energies. The slice was reconstructed by using the mixed absorptionrefraction approach, which requires the acquisition of only one input image per viewing angle [23]. The method was first developed for grating interferometry: however, since the dependence of the signal upon the phase variations is the same for the two techniques (the signal is to first approximation proportional to the refraction angle), the method is equally applicable to the EI setup. The values reconstructed in each pixel are a well-defined linear combination of the absorption and refraction properties of the imaged material [23]: the absorption signal is visible in the bulk regions of the sample, while the refraction one is predominant at the external and internal boundaries of the details in the fossil.

\section{Conclusions}

Our results show that, by going beyond the capabilities of conventional extended X-ray sources, the EI principle can push the limits of the achievable phase sensitivity when implemented with highly coherent SR beams. The unprecedented angular resolution of a few nanoradians we have demonstrated is about one order of magnitude higher than results previously achieved with other XPCi methods.

The technique is very flexible, as demonstrated by its applicability at different synchrotron beamlines, with different detectors and at both very low $(12 \mathrm{keV})$ and very high $(85 \mathrm{keV}) \mathrm{X}$-ray energies. It can therefore be applied over a very broad range of energies, which makes it very promising for the imaging of a variety of samples of different size and composition, in various fields of application, including biology, medicine, materials science etc.

The setup is fairly simple and has relaxed requirements in terms of stability, which makes it very suitable to both quantitative phase retrieval and computed tomography.

\section{Acknowledgments}

The authors would like to thank all personnel at beamlines I13 at Diamond and ID17 at the ESRF. This work is supported by the U.K. Engineering and Physical Sciences Research Council (Grants EP/G004250/1, EP/I022562/1 and EP/I021884/1).

\section{References}

[1] U. Bonse and M. Hart, An X-ray interferometer, Appl. Phys. Lett. 6 (1965) 155.

[2] A. Snigirev, I. Snigireva, V. Kohn, S. Kuznetsov and I. Schelokov, On the possibilities of X-ray phase contrast microimaging by coherent high-energy synchrotron radiation, Rev. Sci. Instrum. 66 (1995) 5486. 
[3] T.J. Davis, D. Gao, T.E. Gureyev, A.W. Stevenson and S.W. Wilkins, Phase-contrast imaging of weakly absorbing materials using hard X-rays, Nature 373 (1995) 595.

[4] A. Momose et al., Demonstration of X-ray Talbot interferometry, Jpn. J. Appl. Phys. 42 (2003) L866.

[5] P.C. Diemoz, A. Bravin and P. Coan, Theoretical comparison of three X-ray phase-contrast imaging techniques: propagation-based imaging, analyzer-based imaging and grating interferometry, Opt. Express 20 (2012) 2789.

[6] P.C. Diemoz, A. Bravin, M. Langer and P. Coan, Analytical and experimental determination of signal-to-noise ratio and figure of merit in three phase-contrast imaging techniques, Opt. Express 20 (2012) 27670.

[7] E. Castelli et al., Mammography with synchrotron radiation: first clinical experience with phase-detection technique, Radiology 259 (2011) 684.

[8] G. Schulz et al., High-resolution tomographic imaging of a human cerebellum: comparison of absorption and grating-based phase contrast, J. Roy. Soc. Interface 7 (2010) 1665.

[9] P. Coan et al., Characterization of osteoarthritic and normal human patella cartilage by computed tomography X-ray phase-contrast imaging: a feasibility study, Invest. Radiol. 45 (2010) 437.

[10] Y. Zhao et al., High-resolution, low-dose phase contrast X-ray tomography for 3D diagnosis of human breast cancers, PNAS 109 (2012) 18290.

[11] A. Olivo et al., An innovative digital imaging set-up allowing a low-dose approach to phase contrast applications in the medical field, Med. Phys. 28 (2001) 1610.

[12] A. Olivo and R.D. Speller, A coded-aperture approach allowing X-ray phase contrast imaging with conventional sources, Appl. Phys. Lett. 91 (2007) 074106.

[13] A. Olivo and R.D. Speller, Modelling of a novel X-ray phase contrast imaging technique based on coded apertures, Phys. Med. Biol. 52 (2007) 6555.

[14] P.R.T. Munro, K. Ignatyev, R.D. Speller and A. Olivo, Design of a novel phase contrast X-ray imaging system for mammography, Phys. Med. Biol. 55 (2010) 4169.

[15] A. Olivo, P.C. Diemoz and A. Bravin, Amplification of the phase contrast signal at very high X-ray energies, Opt. Lett. 37 (2012) 915.

[16] P.R.T. Munro, K. Ignatyev, R.D. Speller and A. Olivo, The relationship between wave and geometrical optics models of coded aperture type X-ray phase contrast imaging systems, Opt. Express 18 (2010) 4103.

[17] C. Ponchut et al., Photon-counting X-ray imaging at kilohertz frame rates, Nucl. Instrum. Meth. A 576 (2007) 109.

[18] B.L. Henke, E.M. Gullikson and J.C. Davis, X-ray interactions: photoabsorption, scattering, transmission, and reflection at $E=50-30000 \mathrm{eV}, Z=1-92$, Atom. Data Nucl. Data 54 (1993) 181.

[19] A. Bravin et al., Comparison between a position sensitive germanium detector and a taper optics CCD “FRELON" camera for diffraction enhanced imaging, Nucl. Instrum. Meth. A 510 (2003) 35.

[20] P.C. Diemoz et al., X-ray phase-contrast imaging with nanoradian angular resolution, Phys. Rev. Lett. 110 (2013) 138105.

[21] P. Modregger et al., Sensitivity of X-ray grating interferometry, Opt. Express 19 (2011) 18324.

[22] F. Pfeiffer et al., High-resolution brain tumor visualization using three-dimensional X-ray phase contrast tomography, Phys. Med. Biol. 52 (2007) 6923.

[23] P.C. Diemoz et al., A simplified approach for computed tomography with an X-ray grating interferometer, Opt. Express 19 (2011) 1691. 